\title{
SPATIAL DAMAGE IDENTIFICATION IN COMPOSITE PLATES USING MULTIWAVELETS
}

\author{
Andrzej Katunin \\ Institute of Fundamentals of Machinery Design, Silesian University of Technology \\ Gliwice, Poland \\ andrzej.katunin@polsl.pl
}

\begin{abstract}
The wavelet transform is one of the most effective tools for many tasks concerning signal and image processing, however it is difficult to obtain all of the necessary properties in one scalar wavelet. This leads to the development of new types of transforms such as a multiwavelet transform, which possesses more than one scaling and wavelet function and makes a possibility to combine these functions in order to obtain necessary properties. In the present study the CL2, LV and DGHM multiwavelets were used for an identification of spatial damage in a composite plate based on the analysis of its modal shapes. The obtained results show that some properties of the multiwavelet transform may improve the damage identification algorithm and replace the classical wavelet-based methods.
\end{abstract}

Keywords: multiwavelets, multiwavelet transform, damage identification, composite plate

\section{Introduction}

The wavelet-based algorithms gain their popularity primarily by their high sensitivity to singularities and non-monotonicities in the analyzed signals. They found an application in signal and image de-noising studies [1,2], pattern recognition [3], numerical algorithms for the approximation of functions [4] or solving integral and differential equations [5-7], etc. Wavelets also found wide application in the structural diagnostics (SD) and structural health monitoring (SHM) problems, i.e. in the detection, localization and identification of external and internal damages in the structural elements used in machinery. The algorithms in the area of structural diagnostics presented to-date mainly based on continuous wavelet transform (CWT) and have been applied to the beams and plates by using various wavelet functions [8-14]. These algorithms are based on the analysis of displacements of modal shapes of vibration. The main advantages of these algorithms are the possibility of precise localization of damage and the fact that the algorithms do not require the model or experimental data of an undamaged structure.

The results of studies carried out by the author over the last few years [15-17] show that the application of the discrete wavelet transform (DWT) is more suitable both for $1 \mathrm{D}$ and $2 \mathrm{D}$ problems due to its best sensitivity and better computational efficiency in comparison with CWT and other related transforms used in SD. 
Moreover, it was proven that the B-spline wavelets provide the best results in SD problems in comparison with other compactly supported wavelets both for $1 \mathrm{D}$ and 2D cases. However, the classic DWT-based algorithm produces some unwanted artefacts in the resulting patterns, e.g. the boundary effect, which causes an increase of detail coefficients on the boundaries of a pattern. The computational methods of its reduction [18] help to solve the problem partially, i.e. the diagnostics of boundary regions of the investigated structure is still not an easy task. Another problem occurs with the identification of spatial damages: the DWT-based algorithm presented in [16] identifies only the boundaries of the damage.

In order to avoid these problems, the discrete multiwavelet transform (DMWT) could be used. The main difference of DMWT regarding to DWT is that the first one uses vector-valued scaling and wavelet functions, which consist of at least two scalar functions. This fact causes that there are more resulting sets of coefficients (usually 4), unlike in the case of scalar wavelets, where only two sets (approximation and detail coefficients) could be obtained. The DMWT creates new possibilities for the signal processing: the multiwavelets could be constructed in such a way that their properties will exactly match the necessities of the investigated problem. These facts cause that the DMWT and multiwavelets improve existed image processing techniques [19], used in the algorithms of gear fault detection [20], in development of new numerical algorithms [21], analysis of geomagnetic pulsations [22] and many others.

In this paper the DMWT was used for the spatial damage identification in a composite plate. The study was performed based on numerical data of modal shapes of vibration of a damaged plate. The vibration data was analysed using three multiwavelets: the Chui-Lian multiwavelet with effective support of 2 (CL2), the Lebrun-Vetterli multiwavelet and the Donovan-Geronimo-Hardin-Massopust (DGHM) multiwavelet. The new DMWT-based damage identification algorithm was developed and presented. The obtained results were compared with results of DWT-based algorithm proposed in [13] and the advantages of the multiwavelet approach were briefly discussed.

\section{Multiwavelets and DMWT}

The multiwavelet theory was introduced by Vasily Strela in his $\mathrm{PhD}$ thesis [23]. Similarly as in the case of scalar wavelets, the multiwavelets are based on the multi-resolution analysis (MRA) with an extension to general dimension $r \in \mathbf{N}$. In DMWT the scaling and wavelet functions are replaced by vector-valued analogues: the vector of scaling functions $\Phi(x)=\left[\phi_{1}(t), \ldots, \phi_{r}(t)\right]^{T}$ and the vector of wavelet functions $\Psi(x)=\left[\psi_{1}(t), \ldots, \psi_{r}(t)\right]^{T}$, which constitutes Riesz bases in $L^{2}(\mathbf{R})^{r}$. Following the statements of Strela in [23] $r$ could be arbitrary large, however the commonly used multiwavelets are primarily with $r=2$. Following the definition of 
MRA of $L^{2}$ the closed functional subspaces $V_{j}$ could be presented in bi-infinite sequential order:

$$
\ldots V_{2} \subset V_{1} \subset V_{0} \subset V_{-1} \subset V_{-2} \ldots
$$

with properties of stability and orthogonality (the detailed description of properties and conditions of multiwavelet MRA could be found in [24]). All of the functions in $\Phi(x)$ and $\Psi(x)$ are in $V_{0} \subset V_{1}$, where $V_{1} \equiv \operatorname{span}\left\{\sqrt{2} \phi_{i}(2 x-k)\right\}, 1 \leq i \leq r, k \in \mathbf{Z}$. The two-scale dilatation and wavelet equations have the form:

$$
\begin{aligned}
& \Phi(x)=\sqrt{2} \sum_{k=0}^{n-1} H_{k} \Phi(2 x-k), \\
& \Psi(x)=\sqrt{2} \sum_{k=0}^{n-1} G_{k} \Psi(2 x-k),
\end{aligned}
$$

where $H_{k}, G_{k} \in l^{2}(\mathbf{z})^{r \times r}$ are the $r \times r$ matrices of filter coefficients of a low-pass filter and a high-pass filter, respectively, for each $k$. A schematic representation of single-level multiwavelet filterbanks was shown in Figure 1.

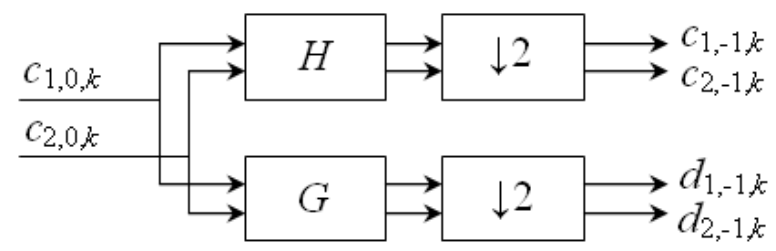

Fig. 1. The scheme of a single-level discrete multiwavelet decomposition

As mentioned before, the DMWT produces more sets of coefficients than DWT. The comparison of 2D subbands of a single-level decomposition for scalar and multiwavelets $(r=2)$ was presented in Figure 2. The symbols ' $\mathrm{H}$ ' and ' $\mathrm{G}$ ' denote the application of a low-pass and high-pass pairs of filters respectively. For example the symbol ' $\mathrm{HG}$ ' denotes low-pass filtering on the rows and high-pass filtering on the columns. The multiwavelets used two channels (see Fig.1), therefore there are two sets of approximation and two sets of detail coefficients. The indices in Figure $2 \mathrm{~b}$ are the number of a given function from $\Phi(x)$ or $\Psi(x)$.

In the case of scalar wavelets the number of vanishing moments often coincides with the length of effective support (e.g. for Daubechies, B-spline wavelets, etc.), which has an influence on their ability of localization of approximation. The multiwavelets could avoid this problem thanks to the combinations of appropriate wavelets. In the following study LV, CV2 and DGHM multiwavelets were selected to analyze the damaged structure. The mentioned multiwavelets were presented graphically in Figure 3. 


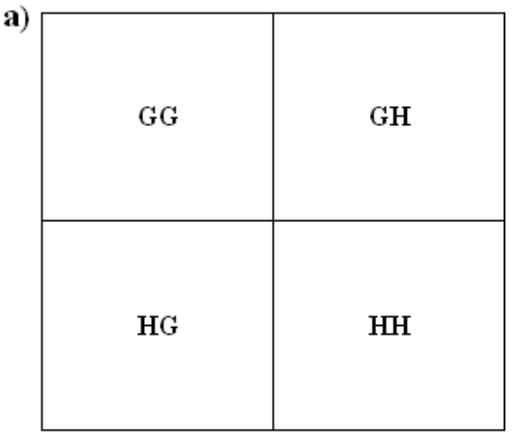

\begin{tabular}{|l|l|l|l|}
\hline $\mathrm{G}_{1} \mathrm{G}_{1}$ & $\mathrm{G}_{1} \mathrm{G}_{2}$ & $\mathrm{G}_{1} \mathrm{H}_{1}$ & $\mathrm{G}_{1} \mathrm{H}_{2}$ \\
\hline $\mathrm{G}_{2} \mathrm{G}_{1}$ & $\mathrm{G}_{2} \mathrm{G}_{2}$ & $\mathrm{G}_{2} \mathrm{H}_{1}$ & $\mathrm{G}_{2} \mathrm{H}_{2}$ \\
\hline $\mathrm{H}_{1} \mathrm{G}_{1}$ & $\mathrm{H}_{1} \mathrm{G}_{2}$ & $\mathrm{H}_{1} \mathrm{H}_{1}$ & $\mathrm{H}_{1} \mathrm{H}_{2}$ \\
\hline $\mathrm{H}_{2} \mathrm{G}_{1}$ & $\mathrm{H}_{2} \mathrm{G}_{2}$ & $\mathrm{H}_{2} \mathrm{H}_{1}$ & $\mathrm{H}_{2} \mathrm{H}_{2}$ \\
\hline
\end{tabular}

Fig. 2. 2D signal subbands after single-level decomposition using: a) DWT, b) DMWT

a)

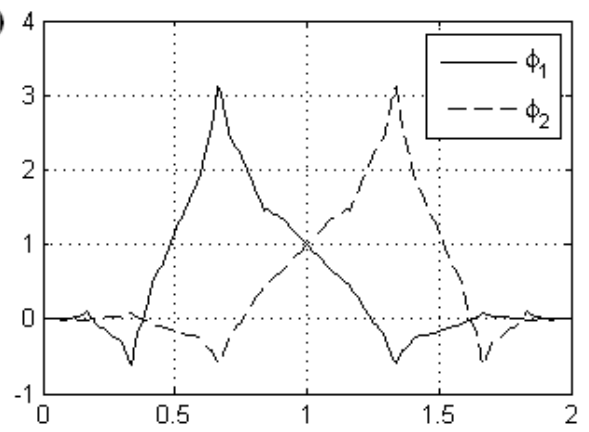

c)

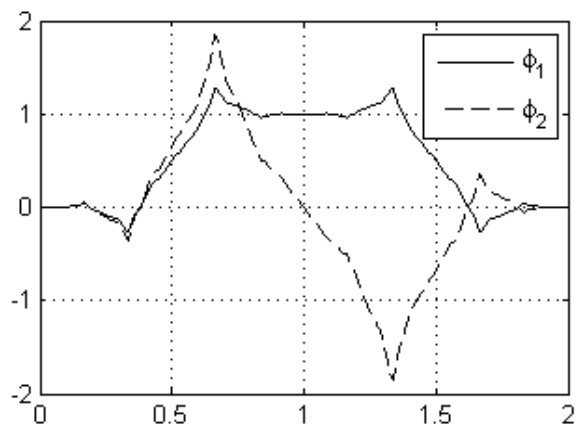

e)

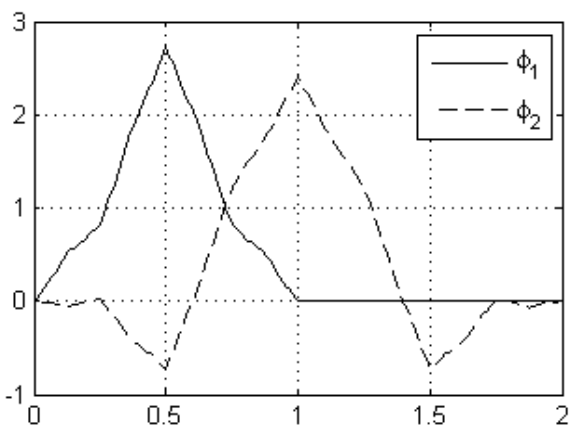

b)

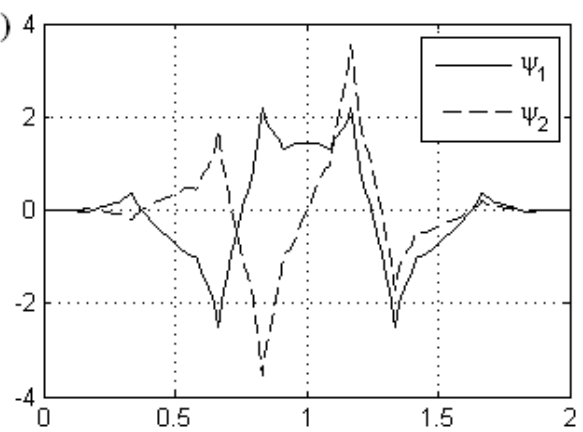

d)
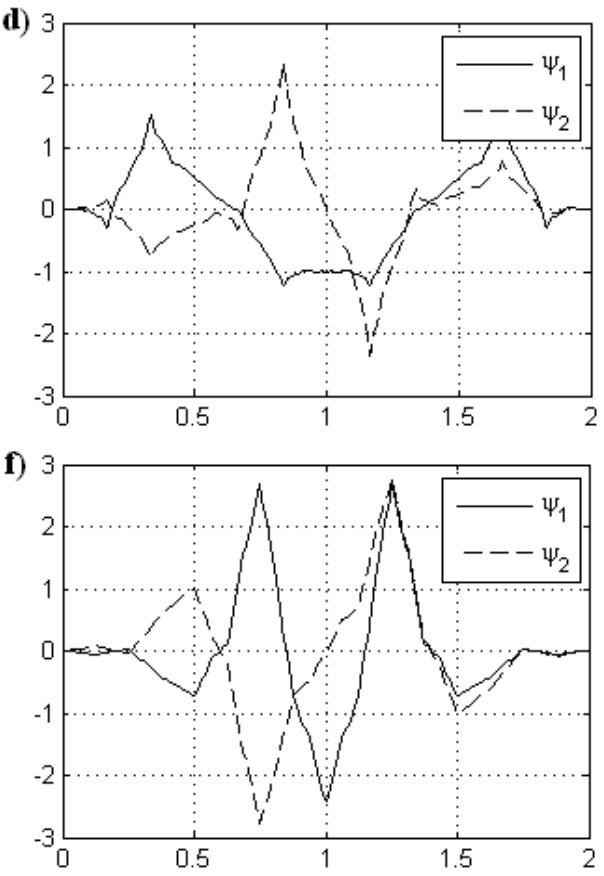

Fig. 3. Scaling and wavelet functions of: a), b) LV multiwavelet, c), d) CL2 multiwavelet and e), f) DGHM multiwavelet 
The first considered multiwavelet is the LV one, introduced in [25]. For this multiwavelet $\phi_{2}(x)$ is the reflection of $\phi_{1}(x)$ about $x=1$ and $\psi_{i}(x)$ are symmetric/ /antisymmetric about $x=1$. The $H_{k}$ and $G_{k}$ are given by the following matrices:

$$
\begin{gathered}
H_{0}=\left[\begin{array}{cc}
0 & \frac{2+\sqrt{7}}{4 \sqrt{2}} \\
0 & \frac{2-\sqrt{7}}{4 \sqrt{2}}
\end{array}\right], H_{1}=\left[\begin{array}{cc}
\frac{3}{4 \sqrt{2}} & \frac{1}{4 \sqrt{2}} \\
\frac{1}{4 \sqrt{2}} & \frac{3}{4 \sqrt{2}}
\end{array}\right], H_{2}=\left[\begin{array}{cc}
\frac{2-\sqrt{7}}{4 \sqrt{2}} & 0 \\
\frac{2+\sqrt{7}}{4 \sqrt{2}} & 0
\end{array}\right], \\
G_{0}=\left[\begin{array}{ll}
0 & \frac{-1}{2} \\
0 & \frac{1}{4}
\end{array}\right], G_{1}=\left[\begin{array}{cc}
\frac{1}{2} & \frac{1}{2} \\
\frac{-\sqrt{7}}{4} & \frac{\sqrt{7}}{4}
\end{array}\right], G_{2}=\left[\begin{array}{cc}
\frac{-1}{2} & 0 \\
\frac{-1}{4} & 0
\end{array}\right] .
\end{gathered}
$$

The CL2 multiwavelet was proposed in [26]. It is symmetric/antisymmetric about $x=1$, the filter coefficients are as follows:

$$
\begin{gathered}
H_{0}=\left[\begin{array}{cc}
\frac{1}{2 \sqrt{2}} & \frac{-1}{2 \sqrt{2}} \\
\frac{\sqrt{7}}{4 \sqrt{2}} & \frac{-\sqrt{7}}{4 \sqrt{2}}
\end{array}\right], H_{1}=\left[\begin{array}{cc}
\frac{1}{\sqrt{2}} & 0 \\
0 & \frac{1}{2 \sqrt{2}}
\end{array}\right], H_{2}=\left[\begin{array}{cc}
\frac{1}{2 \sqrt{2}} & \frac{1}{2 \sqrt{2}} \\
\frac{-\sqrt{7}}{4 \sqrt{2}} & \frac{-\sqrt{7}}{4 \sqrt{2}}
\end{array}\right], \\
G_{0}=\left[\begin{array}{cc}
\frac{1}{2 \sqrt{2}} & \frac{-1}{2 \sqrt{2}} \\
\frac{-1}{4 \sqrt{2}} & \frac{1}{4 \sqrt{2}}
\end{array}\right], G_{1}=\left[\begin{array}{cc}
\frac{-1}{2 \sqrt{2}} & 0 \\
0 & \frac{\sqrt{7}}{2 \sqrt{2}}
\end{array}\right], G_{2}=\left[\begin{array}{cc}
\frac{1}{2 \sqrt{2}} & \frac{1}{2 \sqrt{2}} \\
\frac{1}{4 \sqrt{2}} & \frac{1}{4 \sqrt{2}}
\end{array}\right] .
\end{gathered}
$$

The last considered DGHM multiwavelet was introduced in [27]. $\phi_{1}(x)$ is symmetric about $x=1 / 2$ and $\phi_{2}(x)$ is symmetric about $x=1$. The filter coefficients for this multiwavelet are as follows:

$$
\begin{gathered}
H_{0}=\left[\begin{array}{cc}
\frac{3}{5 \sqrt{2}} & \frac{4}{5} \\
\frac{-1}{20} & \frac{-3}{10 \sqrt{2}}
\end{array}\right], H_{1}=\left[\begin{array}{cc}
\frac{3}{5 \sqrt{2}} & 0 \\
\frac{9}{20} & \frac{1}{\sqrt{2}}
\end{array}\right], \\
H_{2}=\left[\begin{array}{cc}
0 & 0 \\
\frac{9}{20} & \frac{-3}{10 \sqrt{2}}
\end{array}\right], H_{3}=\left[\begin{array}{cc}
0 & 0 \\
\frac{-1}{20} & 0
\end{array}\right],
\end{gathered}
$$




$$
\begin{gathered}
G_{0}=\left[\begin{array}{cc}
\frac{-1}{20} & \frac{-3}{10 \sqrt{2}} \\
\frac{1}{10 \sqrt{2}} & \frac{3}{10}
\end{array}\right], G_{1}=\left[\begin{array}{cc}
\frac{9}{20} & \frac{-3}{10 \sqrt{2}} \\
\frac{9}{10 \sqrt{2}} & \frac{-3}{10}
\end{array}\right], \\
G_{2}=\left[\begin{array}{cc}
\frac{9}{20} & \frac{-3}{10 \sqrt{2}} \\
\frac{9}{10 \sqrt{2}} & \frac{-3}{10}
\end{array}\right], G_{3}=\left[\begin{array}{cc}
\frac{-1}{20} & 0 \\
\frac{-1}{10 \sqrt{2}} & 0
\end{array}\right] .
\end{gathered}
$$

All of the presented multiwavelets are orthogonal and have a compact support of $[0,2]$.

\section{Data preparation, algorithm description and analysis}

In the following section the description of the finite element model definition was presented. The DMWT-based algorithm was introduced and discussed. Based on the numerical data the analysis was performed using three above-mentioned multiwavelets. The results were compared for selected types of multiwavelets and with the results obtained using DWT-based algorithm. Finally the discussion on the obtained results of damage identification was presented.

\subsection{Finite element model and the algorithm}

The finite element model of a composite square plate was prepared in MSC Marc/Mentat commercial software. The dimensions of a plate were as follows: the length of $0.3 \mathrm{~m}$ and the total thickness of $0.015 \mathrm{~m}$. The composite consisted of 12 glass-fiber reinforced polymer layers of the same thickness oriented following the structural formula: $[0 / 60 /-60]_{2 S}$. The material properties were defined as transversal isotropic with the following element values of stiffness matrix: $C_{11}=48.01 \mathrm{GPa}$, $C_{12}=19.98 \mathrm{GPa}, C_{13}=6.592 \mathrm{GPa}, C_{33}=11.42 \mathrm{GPa}, C_{44}=3.533 \mathrm{GPa}, C_{66}=$ $=14.01 \mathrm{GPa}$ and the mass density $\rho=1794 \mathrm{~kg} / \mathrm{m}^{3}$. The clamp boundary condition was defined on the boundaries of a plate. The spatial damage was modelled in the left bottom quarter of a plate in the form of circular exclusion on the top of a plate with the depth of $0.0025 \mathrm{~m}$, which is about $16.7 \%$ of the total thickness of a plate. The dimensions of the exclusion were as follows: a radius of $0.00285 \mathrm{~m}$ and the distances of the centre of an exclusion from the origin along both directions of $0.075 \mathrm{~m}$. The model was meshed using 8-node hexagonal elements with the mesh dimensions of $63 \times 63 \times 6$ elements.

The numerical analysis was performed in order to evaluate the first four modes of vibration. For further studies, the nodal displacements of modal shapes in the direction of thickness axis of the plate were collected. The analyzed cases consisted 
of $64 \times 64$ equidistant measuring points. The number of considered samples must be a power of 2 in order to fulfill the dyadic requirement of DMWT.

The displacements of modal shapes constitute the input data to the DMWT-based algorithm. The proposed algorithm consisted of the following steps: the sets of displacements for every considered modal shape were analyzed separately following the procedure described in the previous section. Only three sets of detail coefficients obtained by low-pass filtering $\left(\mathrm{H}_{1} \mathrm{H}_{2}, \mathrm{H}_{2} \mathrm{H}_{1}\right.$ and $\mathrm{H}_{2} \mathrm{H}_{2}$ - see Fig. $\left.2 b\right)$ were selected for further analysis. As it was noticed, the coefficients magnitudes depend on the magnitudes of displacements, therefore it is necessary to consider more than one modal shape. In order to emphasize the singularities caused by the damage, the absolute values of above-mentioned coefficients were compounded, which constitute $D$-coefficients sets. Such an operation allows to avoid the influence of the damage location and its relation with the currently investigated modal shape.

\subsection{Analysis and comparative studies}

The results of the damage identification obtained following the above-presented procedure were presented in Figure 4. A single-level multiwavelet decomposition was performed following the algorithm presented in section 1 . As it could be noticed, the resulted sets of $D$-coefficients consist the high-magnitude coefficients in the location of a damage. However, in the first two cases, the magnitudes of displacements were not filtered exhaustively and the magnitudes of $D$-coefficients sets contain, besides high values in the damaged region, also high values in the other regions, which may cause difficulties in interpreting of the results (especially in the case of CL2 - Fig. 4b).
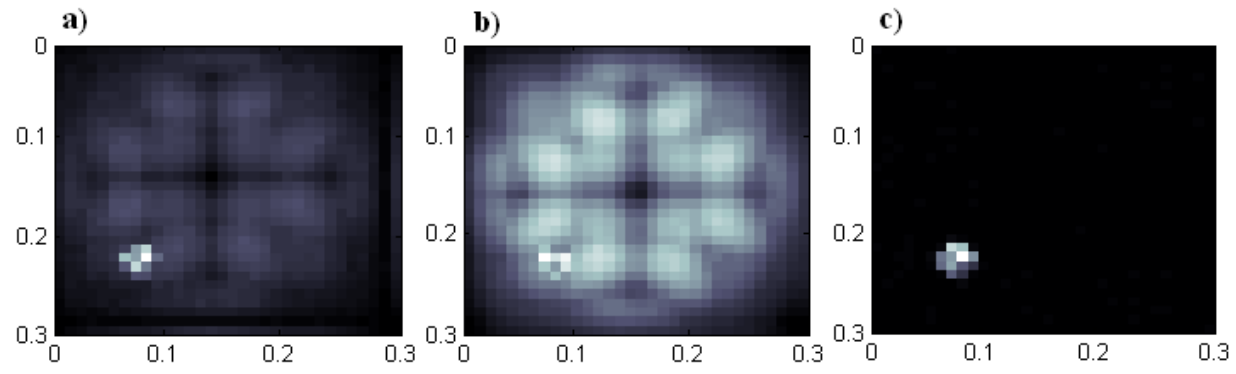

Fig. 4. Results of the damage identification using: a) LV, b) CL2, c) DGHM multiwavelet

In the case of DGHM multiwavelet the damage was clearly detected and localized and the pattern of $D$-coefficients does not contain any additional artefacts. Such results obtained with use of DGHM multiwavelet could be explained by two factors, which influences on the effectiveness of filtering. In spite of other investigated cases of multiwavelets, the DGHM contain wavelets, that are both symmetric. The symmetry of the applied wavelets was studied also in [16] and the same conclusions were obtained for 2D B-spline wavelets. Moreover, $\phi_{1}(x)$ in the DGHM 
multiwavelet has shorter effective support. Following the previous analyzes [16] the length of the effective support influences on the sharpness of obtained results, i.e. the shorter effective support, the sharper results.

In order to compare obtained results with the results obtained using previously developed DWT-based damage identification algorithm the additional study was preformed. As it was reported in [16] the most suitable 2D wavelet used for a spatial damage identification was a six-order 2D B-spline wavelet. The analysis of the same data was performed following this algorithm. The obtained pattern of $D$-coefficients was presented in Figure 5.

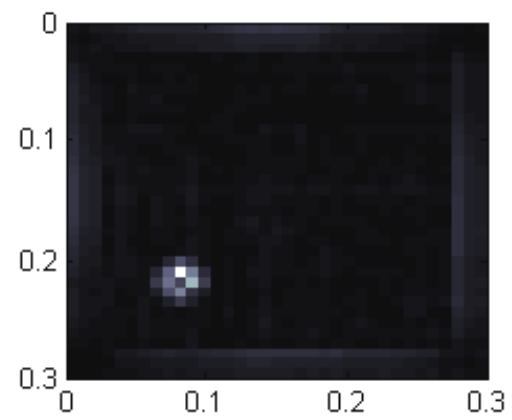

Fig. 5. Results of the damage identification using DWT-based algorithm with 2D six-order B-spline wavelet

As it could be observed, the damage in this case was clearly identified, however the additional artefacts produced by the decomposition procedure presented in the pattern. Moreover, the presence of the boundary effect could be observed (light regions near boundaries), while in the case of DMWT-based algorithm with use of DGHM multiwavelet this effect was presented, however the changes of magnitudes of $D$-coefficients are small and thus the effect is not observable.

\section{Conclusions and discussion}

The paper presented the new damage identification algorithm based on DMWT. Three types of multiwavelets were used for the analysis and the obtained results compared each other and with the results obtained using the previously developed DWT-based damage identification algorithm. Following the presented results it could be concluded that the DMWT-based algorithm with use of DGHM multiwavelet provides the perfect decomposition and filtering, i.e. the modal shapes were completely filtered and only the high-valued $D$-coefficients in the damage location are observable.

The DMWT seems to be a great tool for structural diagnostics and structural health monitoring problems. The developed method could also be applied in the image processing and pattern recognition studies. The DMWT gives an additional unique possibility of almost arbitrary construction of multiwavelets with necessary 
parameters, i.e. the multiwavelets could be constructed for the best identification of damages of a specific type. In the further studies the experimental validation of a method will be performed in laboratory conditions considering a fact that the influence of measurement noise has a great impact on the sensitivity of the method.

\section{Acknowledgements}

The research project was financed by the National Science Centre (Poland) granted according to the decision No. DEC-2011/03/N/ST8/06205.

\section{References}

[1] Donoho D.L., Johnstone I.M., Ideal spatial adaptation via wavelet shrinkage, Biometrica 1994, $81,425-455$.

[2] Donoho D.L., Johnstone I.M., Adapting to unknown smoothness via wavelet shrinkage, J. Amer. Statist. Assoc. 1995, 90, 1200-1224.

[3] Tang Y.Y., Wavelet Theory Approach to Pattern Recognition, World Scientific Publishing, 2nd ed., Series in Machine Perception and Artificial Intelligence, 74, Hong Kong 2009.

[4] Nivergelt Y., Wavelets Made Easy, Springer, Boston 1999.

[5] Nikolaou M., You Y., Use of wavelets for numerical solution of differential equations, [in:] Wavelet Applications in Chemical Engineering, B. Joseph, R. Motard (eds.), Kluwer, 1994, 209-274.

[6] Lakestani M., Razzaghi M., Dehghan M., Solution of nonlinear Fredholm-Hammerstein integral equations by using semiorthogonal spline wavelets, Math. Probl. Eng. 2005, 1, 113-121.

[7] Katunin A., Solution of plane Dirichlet problem using compactly supported 2D wavelet scaling functions, Sci. Res. Inst. Math. Comp. Sci. 2012, 11, 31-40.

[8] Douka E., Loutridis S., Trochidis A., Crack identification in beams using wavelet analysis, Int. J. Solids Struct. 2003, 40, 3557-3569.

[9] Chang C.C., Chen L.W., Damage detection of a rectangular plate by spatial wavelet based approach, Appl. Acoust. 2004, 65, 819-832.

[10] Rucka M., Wilde K., Application of continuous wavelet transform in vibration based damage detection method for beams and plates, J. Sound Vib. 2006, 297, 536-550.

[11] Ziopaja K., Pozorski Z., Damage detection and estimation using wavelet transform, Found. Civ. Env. Eng. 2006, 7, 413-421.

[12] Knitter-Piątkowska A., Pozorski Z., Garstecki A., Application of discrete wavelet transformation in damage detection. Part I: Static and dynamic experiments, Comput. Assist. Mech. Eng. Sci. 2006, 13, 21-38.

[13] Smith C., Akujuobi C.M., Hamory P., Kloesel K., An approach to vibration analysis using wavelets in an application of aircraft health monitoring, Mech. Syst. Signal Pr. 2007, 21, 1255-1272 .

[14] Stępiński T., Uhl T., Staszewski T., Advanced Structural Damage Detection: From Theory to Engineering Applications, John Wiley \& Sons, Chichester 2013.

[15] Katunin A., Identification of multiple cracks in composite beams using discrete wavelet transform, Sci. Probl. Mach. Oper. Maint. 2010, 45, 41-52.

[16] Katunin A., Damage identification in composite plates using two-dimensional B-spline wavelets, Mech. Syst. Signal Pr. 2011, 25, 3153-3167. 
[17] Katunin A., Holewik F., Crack identification in composite elements with non-linear geometry using spatial wavelet transform, Arch. Civ. Mech. Eng. 2013, 13, 287-296.

[18] Katunin A., Reduction of boundary effect during structural damage identification using wavelet transform, Sel. Eng. Probl. 2012, 3, 97-102.

[19] Strela V., Heller P.N., Strang G., Topiwala P., Heil C., The application of multiwavelet filterbanks to image processing, IEEE Trans. Image Proc. 1999, 8, 548-563.

[20] Yuan J., He Z., Zi Y., Gear fault detection using customized multiwavelet lifting schemes, Mech. Syst. Signal Pr. 2010, 24, 1509-1528.

[21] Li M., Zhu J., A multiwavelet Galerkin boundary element method for the stationary Stokes problem in 3D, Eng. Anal. Bound. Elem. 2011, 35, 970-977.

[22] Zanandrea A., Neto C.R., Rosa R.R., Ramos F.F., Analysis of geomagnetic pulsations using multiwavelets spectral and polarization method, Physica A 2000, 283, 175-180.

[23] Strela V., Multiwavelet Theory and Applications, PhD Thesis, Massachusetts Institute of Technology, Cambridge, MA 1996.

[24] Keinert F., Wavelets and Multiwavelets, CRC Press, Boca Ratón 2003.

[25] Lebrun J., Vetterli M., Balanced multiwavelet theory and design, IEEE Trans. Signal Process. 1998, 46, 1119-1125.

[26] Chui C.K., Lian J.-A., A study of orthonormal multi-wavelets, Appl. Numer. Math. 1996, 20, 273-298.

[27] Donovan G.C., Geronimo J.S., Hardin D.P., Massopust P.R., Construction of orthogonal wavelets using fractal interpolation functions, SIAM J. Math. Anal. 1996, 27, 1158-1192. 